\title{
THE NON-AGGREGATED AGGRECAN IN THE HUMAN INTERVERTEBRAL DISC CAN ARISE BY A NON-PROTEOLYTIC MECHANISM
}

\author{
P.J. Roughley, Y. Geng and J.S. Mort*
}

Genetics Unit, Shriners Hospital for Children and McGill University, Montreal, Quebec H3G 1A6, Canada

\begin{abstract}
Analysis of both the aggregated and non-aggregated fractions of aggrecan isolated from adult human intervertebral disc using immunoblotting with antibodies specific for the different domains constituting the aggrecan core protein or atomic force microscopy revealed that many components contained the G1 domain. However, little of the disc aggrecan was able to reform aggregates with hyaluronan, as determined by gel filtration chromatography, suggesting that the G1 domains had been rendered nonfunctional. Since previous studies have shown that disc aggrecan undergoes non-enzymatic glycation with age, the functional effect of such modification was investigated in vitro using bovine aggrecan isolated from young animals. Incubation of monomeric aggrecan with ribose to induce glycation rendered it unable to form complexes with hyaluronan stable to agarose gel electrophoresis or gel filtration chromatography. Similarly, extended treatment of intact proteoglycan aggregate with ribose resulted in destabilisation of the complex with separation of the aggrecan from the hyaluronan. Although it is clear that proteolysis occurs in the intervertebral disc and gives rise to some non-aggregating molecules, a different mechanism is required to explain the presence of many non-aggregating molecules bearing the G1 domain. The products of nonenzymatic glycation of the globular domains of aggrecan would account for this phenomenon and explain why some of the non-aggregating molecules are still large proteoglycans. While such molecules may be retained in the nucleus pulposus, they may be able to diffuse within it, reducing the ability of the tissue to resist compression under asymmetric loading such as bending and ultimately contributing to disc degeneration.
\end{abstract}

Keywords: Aggrecan, intervertebral disc, aggregation, non-enzymatic glycation, hyaluronan interaction.

*Address for correspondence:

John S. Mort

Genetics Unit, Shriners Hospital for Children

1529 Cedar Avenue

Montreal, Quebec H3G 1A6, Canada

Telephone Number: 1-514-282-7166

Fax Number: 1-514-842-5581

E-mail: jmort@shriners.mcgill.ca

\section{List of abbreviations}

A1

A1D1

ADAMTS

CRP

CS1, CS2

DMMB

EGF

G1, G2, G3

GAG

IGD

Ig

KS

MMP

PTR

$\mathrm{V}_{\mathrm{o}}, \mathrm{V}_{\mathrm{t}}$
Proteoglycan aggregate preparation produced from a cartilage extract by caesium chloride density gradient centrifugation under associative conditions (Heinegård, 1972).

Isolated aggrecan prepared by caesium chloride density gradient centrifugation of an A1 fraction under dissociative conditions (Heinegård, 1972).

A disintegrin and metalloproteinase with thrombospondin motifs

Complement-like repeat

Chondroitin sulphate-rich regions in aggrecan

Dimethylmethylene blue

Epidermal growth factor

Aggrecan globular domains 1, 2 and 3

Glycosaminoglycan

Interglobular domain

Immunoglobulin

Keratan sulphate

Matrix metalloproteinase

Proteoglycan tandem repeat

Void and total column volume

\section{Introduction}

The ability of the spine to flexibly support our upright posture is integrally linked to the design of the intervertebral discs that interleave the vertebrae. Each intervertebral disc is composed of an outer annulus fibrosus which is rich in type I collagen and the inner nucleus pulposus which is rich in proteoglycans, primarily the large aggregating proteoglycan, aggrecan. The large size of this proteoglycan and its substitution with numerous sulphated glycosaminoglycan chains provides an immobilised fixed negative charge which endows the tissue with powerful osmotic swelling properties and consequent resistance to static and dynamic compressive forces. Retention of these molecules within the extracellular matrix of the nucleus pulposus is therefore essential to disc function.

The aggrecan molecule consists of a multi-domain $\sim 250 \mathrm{kDa}$ core protein substituted with chondroitin sulphate, keratan sulphate and unsulphated oligosaccharides (Fig. 1a). The N-terminal globular G1domain is composed of an immunoglobulin (Ig) module, which mediates protein/protein interactions with a homologous domain in the ancillary proteoglycan aggregate component, link 
Fig. 1. Aggrecan components in adult human intervertebral disc proteoglycan. (a) Diagram of the aggrecan molecule illustrating the individual components (red) recognised by the antibodies used to probe the western blots. The effect of keratanase and chondroitinase $\mathrm{ABC}$ on the glycosaminoglycan chains is demonstrated on the lower half of the molecule where chondroitin 4-sulphate immunoreactive stubs are generated by the eliminase activity of chondroitinase ABC. (b) The disc proteoglycan preparation was digested with keratanase II and chondroitinase ABC then analysed by western blotting using antisera specific for different regions of the aggrecan molecule. Sample loading was adjusted to overcome differences in antisera affinity and facilitate comparison between the tracks. G1, $2.25 \mu \mathrm{g}$; G2, $7.5 \mu \mathrm{g}$; CS1, $7.5 \mu \mathrm{g}$; G3, $7.5 \mu \mathrm{g}$; CS (chondroitin 4-sulphate stubs), $0.75 \mu \mathrm{g}$. Migration positions of pre-stained molecular weight standards are indicated on the left.

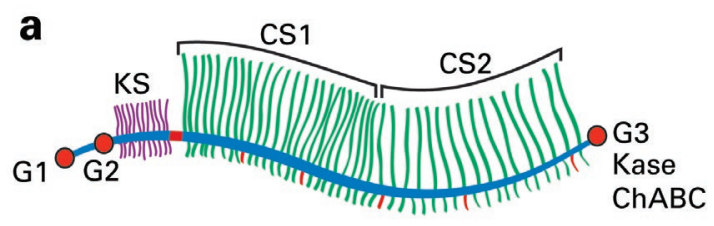

b

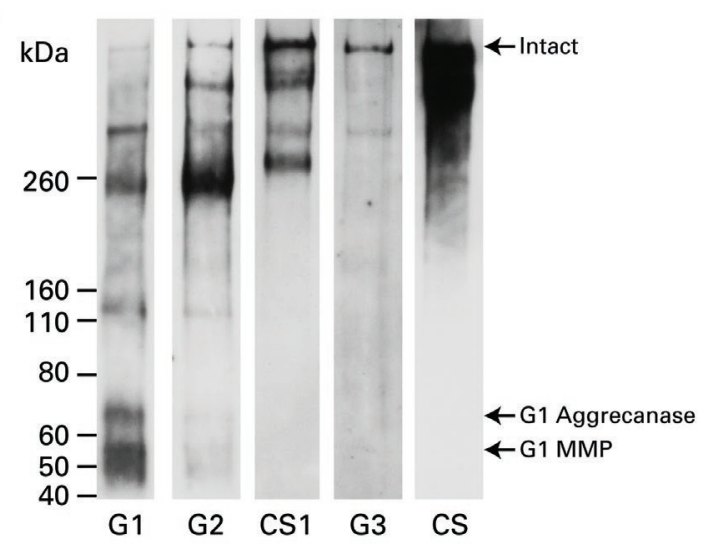

protein, along with two copies of a hyaluronan binding motif also termed the proteoglycan tandem repeat (PTR). Link protein also consists of an Ig module and two PTR motifs. Multiple aggrecan molecules (over 100) bind to a hyaluronic acid filament by way of the PTR motifs in their G1 domains and these complexes are stabilised by interaction with a link protein molecule which binds to the G1 domain and the adjacent region of the hyaluronan strand though its own PTRs. The G1 domain is followed by a short extended interglobular domain (IGD) leading to a second globular domain (G2), which consists of two PTR motifs for which no specific function has been determined so far. The extended nature of the IGD renders it particularly susceptible to proteolytic attack. Such cleavages result in the release of the main glycosaminoglycan-containing component of the aggrecan molecule from its attachment of the hyaluronan filament. The long extended glycosaminoglycan substituted region, which constitutes a major proportion of the protein core, is located between the G2 domain and a C-terminal G3 domain and is divided into three regions. Following the $\mathrm{G} 2$ domain is a region rich in 5-15 kDa keratan sulphate chains. The chondroitin sulphate chains $(\sim 20 \mathrm{kDa})$ are located in two domains designated CS1 and CS2. The human CS1 domain is polymorphic and is composed of 13 to 33 repeats of a 19 residue element, each of which contains two consensus sequences for chondroitin sulphate attachment. The CS2 domain contains the remaining chondroitin sulphate side chains which are somewhat less densely packed than in the CS1 domain. The C-terminal globular domain (G3) results from the expression of a series of exons that encode two epidermal growth factor-like (EGF) repeats, a C-type lectin domain and a complementlike repeat (CRP) domain. Alternate splicing results in the production of variants lacking either or both EGF domains and/or the CRP domain; however the lectin domain is always present. The G3 domain has been shown to interact with tenascins (Aspberg, 2012) and fibulins (Olin et al.,
2001) which may help localise the aggrecan molecules within the disc matrix.

With age and degenerative disease, aggrecan undergoes extensive degradation by multiple proteolytic enzymes particularly of the ADAMTS and MMP families (Sztrolovics et al., 1997). Based on previous studies, intervertebral disc proteoglycans have been divided into two varieties, aggregating and non-aggregating (Melrose and Roughley, 2013). The aggregating proteoglycans are able to interact with hyaluronan and consist of aggrecan along with a smaller amount of another hyalectan, versican. Small leucine-rich repeat proteoglycans such as decorin and biglycan make up some of the non-aggregating proteoglycans, but most of this category is thought to consist of proteolytic products of aggrecan derived from the $\mathrm{KS} / \mathrm{CS} 1 / \mathrm{CS} 2$ regions and lacking the G1 domain. Non-aggregating proteoglycans were found to be more abundant than aggregating proteoglycans in the human nucleus pulposus (Jahnke and McDevitt, 1988).

In this study, adult human intervertebral disc aggrecan was analysed to determine the nature of the non-aggregating components derived from aggrecan. Analysis of the domain structure of the different components indicated that many of the non-aggregated aggrecan molecules retained their G1 domain; however in many cases they appeared to lack the ability to bind hyaluronan. Modification of the G1 domain as a consequence of the formation of advanced glycation end products, which are known to accumulate with ageing, is proposed as the origin of this loss of hyaluronan-binding activity.

\section{Materials and Methods}

\section{Proteoglycan preparation}

Human discs were obtained at autopsy. Lumbar discs were dissected and cut into small fragments ( $13.5 \mathrm{~g}$ wet weight) and extracted in $200 \mathrm{~mL}$ of $4 \mathrm{M}$ guanidinium chloride, 
$0.1 \mathrm{M}$ Tris $\mathrm{HCl}, \mathrm{pH} 7.5$ containing $1 \mathrm{mM}$ EDTA and 4 protease inhibitor cocktail tablets (Roche Diagnostics, Basel, Switzerland) at $4{ }^{\circ} \mathrm{C}$ for $48 \mathrm{~h}$ with stirring. The extract was filtered through glass wool and dialysed exhaustively against $50 \mathrm{mM}$ Tris $\mathrm{HCl}, \mathrm{pH}$ 7.5. Caesium chloride was added to the dialysed extract to a final density of $1.6 \mathrm{~g} /$ $\mathrm{mL}(0.8 \mathrm{~g} / \mathrm{mL}$ of extract $)$ and the mixture centrifuged at 33,000 rpm using a Ti50.2 rotor (Beckman Coulter, Missisauga, Ont., Canada) for $48 \mathrm{~h}$ at $4{ }^{\circ} \mathrm{C}$. The gradient tubes were fractionated into $14 \times 3 \mathrm{~mL}$ fractions with the top fraction consisting of mostly gelatinous material. Fractions were monitored for A280 and glycosaminoglycan (GAG) content. The five lowest density fractions were pooled and dialysed exhaustively against water. A portion $(40 \mathrm{~mL})$ was then dialysed into $0.1 \mathrm{M}$ Tris $\mathrm{HCl}$ for sub-fractionation by gel filtration chromatography and the remainder was freeze-dried (A1-preparation). Bovine foetal epiphyseal cartilage proteoglycan aggregate (A1) and aggrecan (A1D1) were purified as described previously (Roughley and White, 1980).

\section{Separation of aggregated and non-aggregated proteoglycans}

Aliquots ( $5 \mathrm{~mL}$ at $2 \mathrm{mg} / \mathrm{mL}$ ) of the dialysed $\mathrm{A} 1$ preparation were loaded onto a $2.5 \times 120 \mathrm{~cm}$ Sepharose CL2B column and eluted with $0.1 \mathrm{M}$ Tris $\mathrm{HCl}, \mathrm{pH} 7.5$ at a flow rate of $30 \mathrm{~mL} / \mathrm{h}$. Fractions $(5 \mathrm{~mL})$ from 8 such column runs were analysed for GAG content, pooled, dialysed against water, then freeze-dried for further analysis. Aggregated proteoglycan was prepared from fractions eluting at the void volume of the column, and non-aggregated proteoglycan from fractions within the included volume (Fig. 2a).

\section{Glycosaminoglycan assay}

Sulphated GAG content was determined by the dimethylmethylene blue (DMMB) colourimetric assay using chondroitin 6-sulphate (from shark cartilage, SigmaAldrich, St. Louis, MO, USA) as a standard (Farndale et al., 1986).

\section{Sodium dodecyl sulphate polyacrylamide gel electrophoresis and immunoblotting}

Aggrecan samples were digested with keratanase II and chondroitinase $\mathrm{ABC}$ prior to electrophoresis (Durigova et al., 2008). Samples were resolved on Novex 4-12 \% gradient NuPAGE Bis-Tris gels (Invitrogen/ Life Technologies, Carlsbad, CA, USA) under reducing conditions, and transferred to nitrocellulose membranes for immunoblotting, as recommended by the manufacturer. Aggrecan and its degradation products were detected using rabbit polyclonal antibodies recognising the aminoterminal G1 and G2 domains (Sztrolovics et al., 2002), the CS1 region (Roughley et al., 2006) and the carboxyterminal G3 domain (Roughley et al., 2003) of human aggrecan. The mouse monoclonal antibody, 6B, specific for the unsaturated chondroitin-4-sulphate hexasaccharide remaining on the aggrecan core protein following chondroitinase $\mathrm{ABC}$ digestion was kindly provided by $\mathrm{Dr}$. A.R. Poole. Biotinylated anti-rabbit or anti-mouse IgG was used as a secondary antibody, followed by incubation with

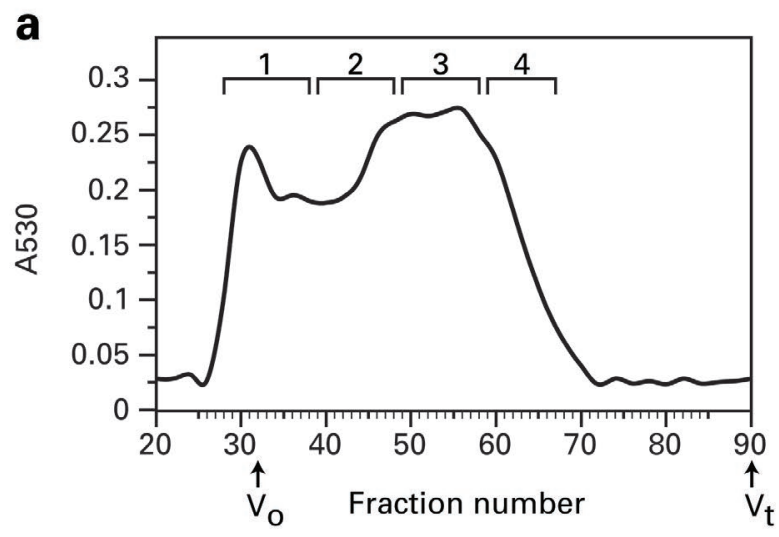

b

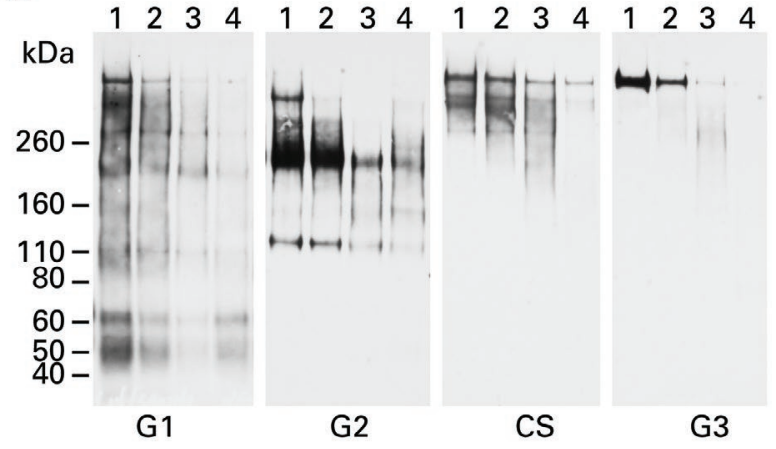

Fig. 2. Size heterogeneity of disc aggrecan components. (a) The disc proteoglycan preparation was fractionated by gel filtration chromatography through Sepharose CL2B and fractions assayed for glycosaminoglycan content. (b) Fractions were combined into four pools as indicated and further analysed by western blotting using antisera specific for different regions of the aggrecan molecule. Sample loading was adjusted to overcome differences in antisera affinity and facilitate comparison between the tracks. G1, $2.25 \mu \mathrm{g} ; \mathrm{G} 2,7.5 \mu \mathrm{g}$; G3, $7.5 \mu \mathrm{g}$; CS (chondroitin 4-sulphate stub), $0.75 \mu \mathrm{g}$. Migration positions of pre-stained molecular weight standards are indicated on the left.

a streptavidin biotinylated horseradish peroxidase complex (Amersham, Amersham, UK). Finally Amersham ECL western blot detection reagents were used to visualise the reactive bands.

\section{Ribose treatment}

Bovine foetal epiphyseal cartilage proteoglycan aggregate (A1) and purified aggrecan (A1D1) at $1 \mathrm{mg} / \mathrm{mL}$ were incubated with various concentrations of D-(-)-ribose (Sigma-Aldrich) for extended periods in phosphate buffered saline (PBS; $10 \mathrm{mM}$ sodium phosphate, $\mathrm{pH} 7.2$, $0.145 \mathrm{M} \mathrm{NaCl}$ ).

\section{Agarose gel electrophoresis}

Aggrecan $(100 \mu \mathrm{g}$ in $100 \mu \mathrm{L}$ of PBS) was treated with up to $0.1 \mathrm{M}$ ribose for 4 days at $37^{\circ} \mathrm{C}$. Samples $(20 \mu \mathrm{L})$ were incubated with or without $2 \mu \mathrm{g}$ of hyaluronan at room temperature for $20 \mathrm{~min}$ and aliquots $(10 \mu \mathrm{L})$ were analysed by $1.2 \%$ agarose gel electrophoresis with Toluidine blue staining (Lee and Cowman, 1994). 

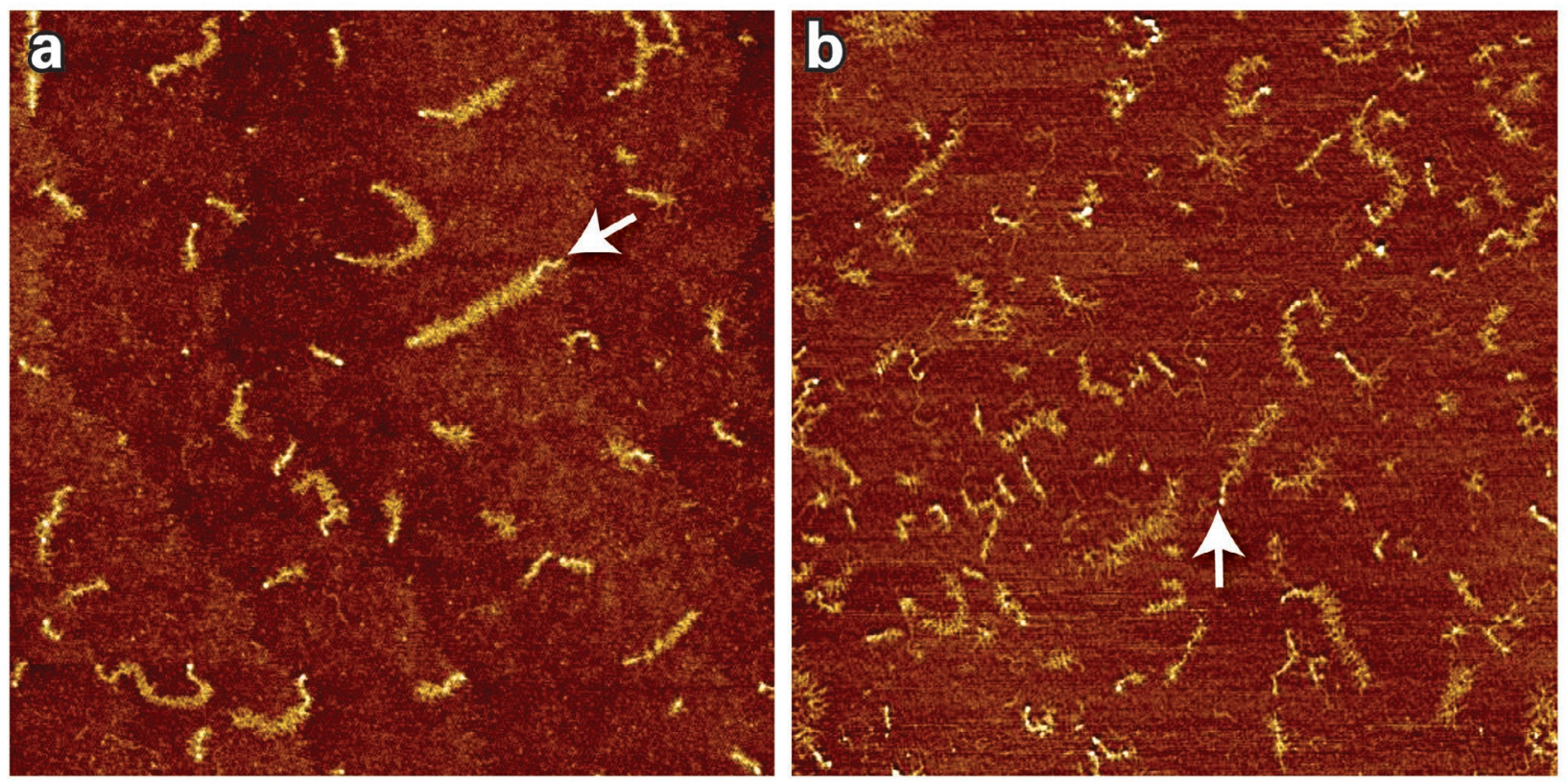

Fig. 3. AFM analysis of disc aggrecan. Tapping mode AFM height images of aggregated (a) and non-aggregated (b) disc proteoglycan are depicted. Globular domains indicative of hyaluronan-binding regions are indicated by arrows.

\section{Reduction and alkylation}

Aggrecan samples $(1 \mathrm{mg} / \mathrm{mL})$ in $4 \mathrm{M}$ guanidinium chloride, $50 \mathrm{mM}$ Tris/ $\mathrm{HCl}, \mathrm{pH} 7.4$ containing $5 \mathrm{mM}$ dithiothreitol were incubated at $37^{\circ} \mathrm{C}$ for $4 \mathrm{~h}$. Iodoacetamide was then added to a final concentration of $15 \mathrm{mM}$ and the mixture left at room temperature overnight. The samples were dialysed into column buffer $(0.1 \mathrm{M}$ Tris/ $\mathrm{HCl} \mathrm{pH} 7.5,50 \mathrm{mM} \mathrm{NaCl})$ for analysis by Sephacryl S-1000 chromatography.

\section{Sephacryl S-1000 chromatography}

Hyaluronan $(20 \mu \mathrm{g})$ was added to aggrecan samples ( $1 \mathrm{mg} /$ $\mathrm{mL}$ ) treated with or without ribose. Samples were loaded onto a 1 x $100 \mathrm{~cm}$ Sephacryl S-1000 column equilibrated with $0.1 \mathrm{M}$ Tris/ $\mathrm{HCl}, \mathrm{pH} 7.5,50 \mathrm{mM} \mathrm{NaCl}$ and eluted with a flow rate of $6 \mathrm{~mL} / \mathrm{h} .1 \mathrm{~mL}$ fractions were collected and analysed for GAG content.

\section{Atomic force microscopy}

Aggregated and non-aggregated proteoglycan preparations (50 $\mu \mathrm{L}$ at $100 \mu \mathrm{g} / \mathrm{mL}$ GAG) were deposited on 3-aminopropyltriethoxysilane (APTES; Sigma-Aldrich) -treated muscovite mica surfaces (SPI Supplies, West Chester, PA, USA) for 20-30 min at room temperature, rinsed gently with MilliQ water and air dried. Samples were imaged using a Nanoscope IIIa Multimode AFM (Veeco, Santa Barbara, CA, USA) in tapping mode at ambient conditions using probe tips with rectangular Si cantilevers (AC240TS-2, Olympus, nominal spring constant $\mathrm{k}=2 \mathrm{~N} / \mathrm{m}$, nominal tip radius $<10 \mathrm{~nm}$ ) (Lee et al., 2013).

\section{Results}

The proteoglycan content of the adult human lumbar discs was extracted under denaturing conditions and, following dialysis into renaturing conditions, purified by caesium chloride density gradient ultracentrifugation. After digestion with keratanase and chondroitinase ABC to reduce the glycosaminoglycan chains to remnant oligosaccharides, the proteoglycan (A1) fraction was analysed by western blotting using antibodies specific for different regions of the aggrecan molecule (Fig. 1b). The G1 domain was present in a wide variety of constituents ranging from the intact core protein to isolated G1 components previously shown to result from MMP or aggrecanase cleavage of the interglobular region (Sztrolovics et al., 1997). The G2 domain was present in all components greater than $100 \mathrm{kDa}$ in size. The G3 domain was predominantly present in the intact aggrecan core protein although a small amount of lower molecular weight material was present. CS1-containing material consisted of the intact molecule and components migrating greater than $200 \mathrm{kDa}$. Staining for the chondroitin 4-sulphate stubs remaining following chondroitinase $\mathrm{ABC}$ digestion mirrored the pattern observed for CS1. Surprisingly there was little evidence for fragments lacking the G1 domain, as would have been expected for intervertebral disc which has been reported to possess a high level of non-aggregating proteoglycans derived by proteolysis of aggrecan.

The intervertebral disc proteoglycan constituents were further resolved into aggregating and non-aggregating components by gel filtration chromatography (Fig. 2). The resulting fractions were collected into four pools. Pool 1 - aggregated proteoglycan; Pools 2-4, - large, medium and small non-aggregated proteoglycans. The different aggrecan domains were analysed in these four proteoglycan pools. The G1 domain predominated in pool 1 but was also observed in the other pools and was represented in all components recognised by the antibodies specific for the other aggrecan components. The G3 domain was predominant in pools 1 and 2, but deficient in pools 3 and 4 as expected if proteolysis occurs from the $\mathrm{C}$-terminus. It should be noted that no very small chondroitin sulphate- 
containing fragments (which would elute close to the $\mathrm{V}$, region) were present in the preparation. Thus it appears that both aggregating and non-aggregating proteoglycans in the intervertebral disc can possess a G1 domain.

Aggregated and non-aggregated intervertebral disc preparations were also imaged directly by atomic force microscopy (Fig. 3). A wide range of size heterogeneity was present in the case of the aggregated proteoglycan samples, where components representing isolated globular regions are seen through to intact aggrecan molecules in which globular regions are clearly visible at both ends of the extended proteoglycan filament. Essentially the same pattern of molecules is observed for the non-aggregated proteoglycans, where $\mathrm{G} 1$ domains are visible in many of the molecules. This finding complements the conclusions reached by western blotting analysis.

To verify that the high abundance of non-aggregated aggrecan was not due to a deficiency of hyaluronan in the disc extract, the ability of the disc proteoglycan preparation to form aggregates with exogenously added hyaluronan was analysed by Sephacryl S1000 chromatography (Fig. 4). When chromatographed in the absence of hyaluronan, most of the GAG-containing material eluted in the included volume. The profile was essentially unchanged when the sample was treated with hyaluronan prior to elution, indicating that the G1 domains in the nonaggregated aggrecan molecules were unable to interact with hyaluronan. Moreover, reduction and alkylation of the proteoglycan preparation prior to analysis had no effect on the size of the smaller aggrecan molecules (Fig. 4), demonstrating that the non-aggregated proteoglycan pools represent single molecules rather than small aggregates.

The above findings indicate that, in contrast to expectations, the non-aggregated fraction of intervertebral disc aggrecan appears to retain the G1 domain but its function is impaired. Since it is well established that nonenzymatic glycation of matrix molecules is a consequence of ageing in connective tissues, the possibility of such chemical modification of the G1 domain was investigated using a model system in which bovine aggrecan was incubated with various concentrations of the reducing sugar ribose at $37{ }^{\circ} \mathrm{C}$ for four days. The ability of the resulting proteoglycan to form aggregates with hyaluronan was analysed by agarose gel electrophoresis (Fig. 5). In the absence of hyaluronan, bovine aggrecan migrates

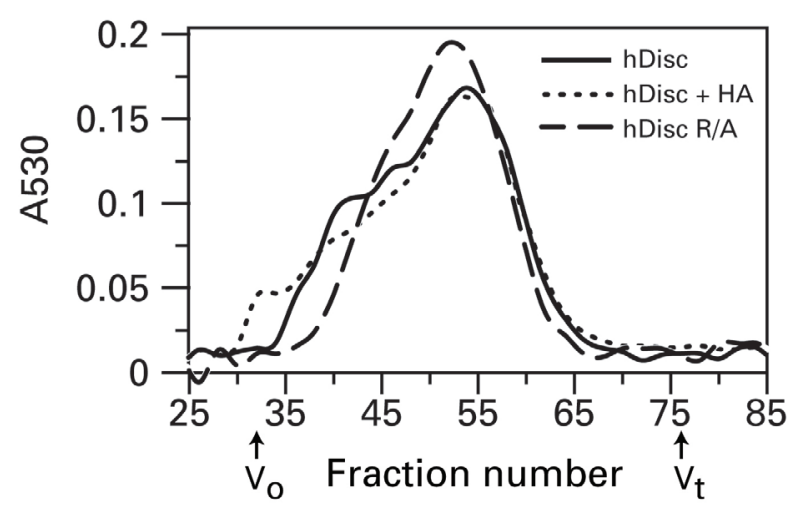

Fig. 4. Lack of interaction of intervertebral disc proteoglycan with hyaluronan. The disc proteoglycan preparation (1 $\mathrm{mg}$ ) was analysed alone or with additional hyaluronan $(20 \mu \mathrm{g})$ by Sephacryl S-1000 chromatography, and the glycosaminoglycan content of the fractions determined. Additional samples were also reduced and alkylated (R/A) prior to analysis. Elution positions for the void volume $\left(\mathrm{V}_{0}\right)$ and total column volume $\left(\mathrm{V}_{\mathrm{t}}\right)$ are indicated.

into the gel as a well-defined band. However in the presence of hyaluronan this migration is retarded due to aggregation and the proteoglycan remains immobilised at the origin, indicating that all of the aggrecan is present in the aggregated form. While incubation in the presence of increasing concentrations of ribose did not alter the migration of the aggrecan, indicating the absence of any catabolic event, this treatment had a dramatic effect on its ability to form aggregates. Based on these studies $100 \mathrm{mM}$ ribose was used in further studies.

The effect of ribose on the ability of aggrecan to form high molecular weight aggregates was confirmed by gel exclusion chromatographic analysis in the presence of $2 \%$ hyaluronan. Extended incubation at $45{ }^{\circ} \mathrm{C}$ in the presence of $100 \mathrm{mM}$ ribose resulted in a substantial decrease in the percentage of the aggrecan that was able to elute at the void volume of the column (Fig. 6a). However, the elution position or the monomer fraction was not altered by the treatment confirming the absence of proteolytic degradation. Thus, non-enzymatic glycation can prevent proteoglycan aggregate formation. However, this does not prove that it could destabilise existing aggregates. To test
Fig. 5. Effect of ribose treatment on the ability of aggrecan to complex with hyaluronan. Bovine aggrecan $(100 \mu \mathrm{g})$ was incubated with the indicated ribose concentrations $(0-100 \mathrm{mM})$ for 4 days at $37^{\circ} \mathrm{C}$. Samples for each ribose concentration were analysed directly or treated with hyaluronan $(2 \mu \mathrm{g})$ at room temperature for $20 \mathrm{~min}$, and $10 \mu \mathrm{g}$ aliquots were then analysed by agarose gel electrophoresis and Toluidine blue staining.

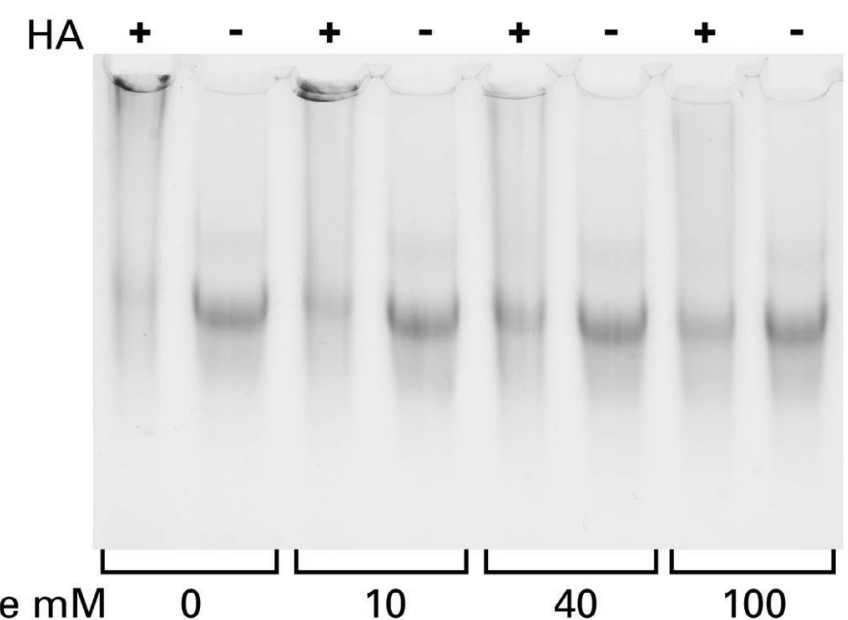


this possibility, a bovine A1 preparation was incubated with $100 \mathrm{mM}$ ribose for 10 weeks at $25^{\circ} \mathrm{C}$. In the absence of ribose about $40 \%$ of the proteoglycan eluted in the aggregated form at the void volume, while prior incubation with ribose resulted in a reduction of the void volume peak to about $10 \%$ of the total. Control experiments investigated possible degrading effects of ribose on hyaluronan under the same conditions. No change in hyaluronan size was seen on agarose gel electrophoresis (data not shown), indicating that dissociation of aggregates observed under conditions favouring non-enzymatic glycation is not due to hyaluronan cleavage but rather modification of the aggrecan G1 domain to impair its interaction with hyaluronan.

\section{Discussion}

It has been reported previously that a large fraction of the proteoglycan isolated from the adult human intervertebral disc is unable to form aggregates with hyaluronan. This has been attributed to proteolytic cleavage of the aggrecan core protein and consequent absence of the hyaluronanbinding G1 domain from many of the fragments. In the present analysis of the non-aggregated fraction of disc proteoglycan using antibodies specific for the different aggrecan domains, most of the non-aggregated fraction appeared to retain immuno-reactive G1 material, suggesting that, rather than being removed by proteolytic action, this region had been rendered non-functional. This effect was reproduced in vitro by generation of glycation products, suggesting that loss of the ability of the aggrecan to form stable complexes may be a consequence of chemical modification of the G1 domain as occurs during non-enzymatic glycation.

Non-enzymatic glycation occurs through a complex series of reactions, originating with the interaction of the side chains of lysine and arginine residues in proteins with the carbonyl group on reducing sugars, resulting in the generation of Maillard products (Vistoli et al., 2013) and is usually considered to be a consequence of natural ageing. In connective tissues many components are susceptible to derivatisation, in particular collagen and aggrecan. Various sugars have been shown to introduce pentosidine crosslinks into proteins (Sell and Monnier, 1989) and the content of this product can be used as a measure of the extent of non-enzymatic glycation. The rate of crosslink formation by pentoses is greater than by hexoses, with ribose being the most reactive monosaccharide in vitro (Dyer et al., 1991). The higher reactivity of ribose over glucose is thought to relate to the presence of a higher proportion of the reactive acyclic isoform in the former (Cho et al., 2007). In vivo, it is unlikely that ribose concentrations are sufficient to contribute substantially to pentosidine formation and glucose is probably the major monosaccharide involved. An increase in Maillard products, as exemplified by pentosidine, has been reported for cartilage collagen (Verzijl et al., 2000) and aggrecan (Verzijl et al., 2001) and a similar increase in abundance of pentosidine derivatisation was observed in human intervertebral disc collagen and aggrecan with age

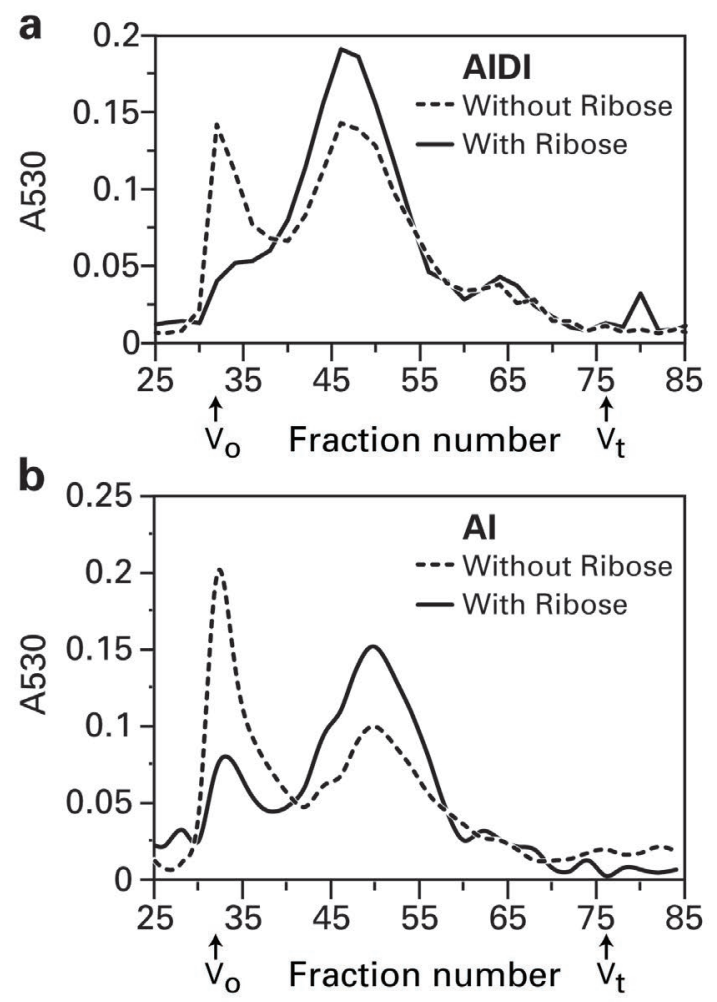

Fig. 6. Effect of ribose treatment on aggregation of aggrecan. (a) Isolated bovine aggrecan (A1D1, $1 \mathrm{mg}$ ) was treated with or without $100 \mathrm{mM}$ ribose for three days at $45^{\circ} \mathrm{C}$. Following addition of $20 \mu \mathrm{g}$ of hyaluronan, samples were analysed by Sephacryl S-1000 chromatography and the glycosaminoglycan content of the resulting fractions determined. (b) Bovine proteoglycan aggregate (A1, $1 \mathrm{mg}$ ) was treated with or without $100 \mathrm{mM}$ ribose for 2.5 months at room temperature. Samples were analysed directly by Sephacryl S-1000 chromatography and the glycosaminoglycan content of the resulting fractions determined. Elution positions for the void volume $\left(\mathrm{V}_{0}\right)$ and total column volume $\left(\mathrm{V}_{\mathrm{t}}\right)$ are indicated.

(Sivan et al., 2006a). Due to the relatively long lifetime of aggrecan in human articular cartilage (Sivan et al., 2006b), the accumulation of modified lysine side chains would be expected to have functional consequences for the interaction of the aggrecan G1 domain with hyaluronan, since lysine residues have been shown be contribute to this process (Hardingham et al., 1976). In order to mimic this process, in vitro, in a feasible time period, the reactive sugar ribose was incubated with functional bovine aggrecan at slightly higher than physiological temperature. Under these conditions, the ability to aggregate with hyaluronan was lost in 3-4 days. In contrast 2.5 months of incubation was required (at room temperature) to destabilise a pre-existing aggrecan/hyaluronan/link protein complex, indicating that the lysine residues mediating these interactions are slowly accessible to modification while in the disc tissue.

Our findings that the G1-domain is retained in the non-aggregated fraction of intervertebral aggrecan, and that "ageing" of aggrecan and aggrecan/hyaluronan/link protein complexes in vitro by incubation with ribose leads 
to loss of the ability of G1-containing aggrecan molecules form stable complexes with hyaluronan, suggest that nonenzymatic glycation reactions in the intervertebral disc can have a detrimental functional effect on the endogenous aggrecan molecules which increases with age.

Aggrecan molecules isolated from intervertebral disc varied widely in size indicating that they had undergone extensive proteolysis; however, there was little evidence for proteoglycan fragments lacking the G1 domain. As appears to be the case in cartilage, it is likely that these products are lost from the tissue by diffusion due to their small size. However, although the remaining G1-containing aggrecan molecules are retained in the disc tissue, they would not be as constrained as fully functional hyaluronan-associated complexes. As a consequence, these large proteoglycans, while restricted to the nucleus pulposus, may be able to migrate within it, reducing the ability of the tissue to resist compression under asymmetric loading, such as bending, and ultimately contributing to disc degeneration.

While in the present study ribose was used to induce glycation reactions with aggrecan because of its enhanced reactivity relative to other reducing sugars (Sell and Monnier, 1989), glucose may play a more prominent role in vivo. The increased abundance of glucose, in the circulation in diabetes, renders it a major hazard and protein glycation is an unfortunate consequence of the disease leading to vascular insufficiency (Harcourt et al., 2013). In addition, as the present study would suggest, increased intervertebral disc degeneration is also a consequence of diabetes (Illien-Junger et al., 2013; Robinson et al., 1998).

\section{Conclusions}

The human intervertebral disc is a sealed environment where the products of proteolytic degradation of aggrecan are retained until cleaved into small products. The previous finding that the majority of the proteoglycans extracted from intervertebral disc are unable to form complexes with hyaluronan was confirmed in this study, but the results suggest that, in addition to proteolytic degradation, non-enzymatic glycation of the aggrecan core protein contributes to their generation. Non-enzymatic glycation results in reduction of the ability of the G1 domain to bind to hyaluronan, and lowers the effective size of the glycosaminoglycan-containing molecules allowing them to migrate within the tissue extracellular matrix, impairing the stability of the intervertebral disc to bending motions and likely contributing to disc degeneration. These findings provide argument for the use of inhibitors of advancedglycation end products as a long term strategy to promote disc health.

\section{Acknowledgements}

This work was supported by the Canadian Institutes for Health Research (MOP106588) and the Shriners of North America. We thank Dr. Richard H. Pearce for providing the disc materials. We are most grateful to Fei Liang and Professors Christine Ortiz and Alan Grodzinsky
(Massachusetts Institute of Technology) for the atomic force microscopy.

\section{References}

Aspberg A (2012) The different roles of aggrecan interaction domains. J Histochem Cytochem 60: 987-996.

Cho SJ, Roman G, Yeboah F, Konishi Y (2007) The road to advanced glycation end products: a mechanistic perspective. Curr Med Chem 14: 1653-1671.

Durigova M, Roughley PJ, Mort JS (2008) Mechanism of proteoglycan aggregate degradation in cartilage stimulated with oncostatin M. Osteoarthritis Cartilage 16: 98-104.

Dyer DG, Blackledge JA, Thorpe SR, Baynes JW (1991) Formation of pentosidine during nonenzymatic browning of proteins by glucose. Identification of glucose and other carbohydrates as possible precursors of pentosidine in vivo. J Biol Chem 266: 11654-11660.

Farndale RW, Buttle DJ, Barrett AJ (1986) Improved quantitation and discrimination of sulphated glycosaminoglycans by use of dimethylmethylene blue. Biochim Biophys Acta 833: 173-177.

Harcourt BE, Penfold SA, Forbes JM (2013) Coming full circle in diabetes mellitus: from complications to initiation. Nat Rev Endocrinol 9: 113-123.

Hardingham TE, Ewins RJ, Muir H (1976) Cartilage proteoglycans. Structure and heterogeneity of the protein core and the effects of specific protein modifications on the binding to hyaluronate. Biochem J 157: 127-143.

Heinegård D (1972) Extraction, fractionation and characterization of proteoglycans from bovine tracheal cartilage. Biochim Biophys Acta 285: 181-192.

Illien-Junger S, Grosjean F, Laudier DM, Vlassara H, Striker GE, Iatridis JC (2013) Combined anti-inflammatory and anti-age drug treatments have a protective effect on intervertebral discs in mice with diabetes. PLoS One 8: e64302.

Jahnke MR, McDevitt CA (1988) Proteoglycans of the human intervertebral disc. Electrophoretic heterogeneity of the aggregating proteoglycans of the nucleus pulposus. Biochem J 251: 347-356.

Lee HG, Cowman MK (1994) An agarose gel electrophoretic method for analysis of hyaluronan molecular weight distribution. Anal Biochem 219: 278287.

Lee HY, Han L, Roughley PJ, Grodzinsky AJ, Ortiz C (2013) Age-related nanostructural and nanomechanical changes of individual human cartilage aggrecan monomers and their glycosaminoglycan side chains. J Struct Biol 181: 264-273.

Melrose J, Roughley P (2013) Proteoglycans of the intervertebral disc. In: Intervertebral Disc (Shapiro IM, Risbud MV, eds), Springer Verlag, Vienna, pp 53-77.

Olin AI, Mörgelin M, Sasaki T, Timpl R, Heinegård D, Aspberg A (2001) The proteoglycans aggrecan and versican form networks with fibulin-2 through their lectin domain binding. J Biol Chem 276: 1253-1261.

Robinson D, Mirovsky Y, Halperin N, Evron Z, Nevo Z (1998) Changes in proteoglycans of intervertebral disc 
in diabetic patients. A possible cause of increased back pain. Spine (Phila Pa 1976) 23: 849-855.

Roughley PJ, White RJ (1980) Age-related changes in the structure of the proteoglycan subunits from human articular cartilage. J Biol Chem 255: 217-224.

Roughley PJ, Barnett J, Zuo F, Mort JS (2003) Variations in aggrecan structure modulate its susceptibility to aggrecanases. Biochem J 375: 183-189.

Roughley PJ, Melching LI, Heathfield TF, Pearce RH, Mort JS (2006) The structure and degradation of aggrecan in human intervertebral disc. Eur Spine J 15 (Suppl 15): 326-332.

Sell DR, Monnier VM (1989) Structure elucidation of a senescence cross-link from human extracellular matrix. Implication of pentoses in the aging process. J Biol Chem 264: 21597-21602.

Sivan SS, Tsitron E, Wachtel E, Roughley P, Sakkee N, van der HF, Degroot J, Maroudas A (2006a) Age-related accumulation of pentosidine in aggrecan and collagen from normal and degenerate human intervertebral discs. Biochem J 399: 29-35.

Sivan SS, Tsitron E, Wachtel E, Roughley PJ, Sakkee N, van der Ham F, DeGroot J, Roberts S, Maroudas A (2006b) Aggrecan turnover in human intervertebral disc as determined by the racemization of aspartic acid. J Biol Chem 281: 13009-13014.

Sztrolovics R, Alini M, Roughley PJ, Mort JS (1997) Aggrecan degradation in human intervertebral disc and articular cartilage. Biochem J 326: 235-241.

Sztrolovics R, White RJ, Roughley PJ, Mort JS (2002) The mechanism of aggrecan release from cartilage differs with tissue origin and the agent used to stimulate catabolism. Biochem J 362: 465-472.

Verzijl N, Degroot J, Thorpe SR, Bank RA, Shaw JN, Lyons TJ, Bijlsma JW, Lafeber FP, Baynes JW, TeKoppele JM (2000) Effect of collagen turnover on the accumulation of advanced glycation end products. J Biol Chem 275: 39027-39031.

Verzijl N, Degroot J, Bank RA, Bayliss MT, Bijlsma JW, Lafeber FP, Maroudas A, TeKoppele JM (2001) Agerelated accumulation of the advanced glycation endproduct pentosidine in human articular cartilage aggrecan: the use of pentosidine levels as a quantitative measure of protein turnover. Matrix Biol 20: 409-417.

Vistoli G, De MD, Cipak A, Zarkovic N, Carini M, Aldini G (2013) Advanced glycoxidation and lipoxidation end products (AGEs and ALEs): an overview of their mechanisms of formation. Free Radic Res 47(Suppl 1): 3-27.

\section{Discussion with Reviewers}

G. Dodge: Can the authors provide some rationale why D-ribose was the target molecule of interest in this study given that the levels of this sugar in the IVD would not be expected to be attained under physiological conditions?
Authors: The rate of crosslink formation by pentoses is greater than by hexoses, with ribose being the most reactive monosaccharide in vitro (Dyer et al., 1991, text reference). As with previous in vitro glycation studies, D-ribose was used as the target molecule to allow AGE development on a feasible time scale. In vivo, it is unlikely that ribose concentrations are sufficient to contribute substantially to pentosidine formation and glucose is probably the major monosaccharide involved, fortunately for human tissue welfare, at a much slower rate.

G. Dodge: Why is D-glucose less effective than D-ribose for these induced modifications of aggrecan? The implication for diabetic patients is quite clear. I should point out that Metformin, a commonly used diabetic medication, seems to spare collagen from AGE type modifications thus may also have a similar sparing effect with aggrecan.

Authors: The higher reactivity of ribose over glucose is thought to relate to the presence of a higher proportion of the reactive acyclic isoform in the former (Cho et al., 2007, text reference). The point regarding the biguanide, Metformin, is well taken. We agree that the sparing effect seen on collagen should also translate to aggrecan. It will be interesting to see if treatment of type II diabetic patients with Metformin can be linked to a decrease in disc pathology already associated with this disease.

J. Melrose: Why didn't the study include PG from tissues of different ages? This would have strengthened the conclusions that these molecules occur in ageing and related to the modification they report.

Authors: It has been well established that the proportion of non-aggregating fragments of aggrecan increases with age, but it is not known whether the presence of G1 regions indicative of non-enzymatic glycation is also age dependent. Based on the known age-related increase in pentosidine in disc aggrecan, we would expect this to be the case. However, as indicated by the reviewer, direct study of disc proteoglycans from tissue of different ages would be required to confirm this.

J. Melrose: Also of interest would be the level of glycation of the starting material and that which resulted from the in vitro approach.

Authors: We agree that more information on glycation levels would have enhanced the present study; however, deriving these data is not a trivial task. In the tissue, multiple glycation products are present and there is no single assay to detect them all. Pentosidine is one such product but its levels need not reflect total modified lysine. In addition, pentosidine quantitation would give no information as to whether modification of the lysine residues would have functional significance. To address this issue we chose a functional rather than a quantitative assay to determine whether proteoglycan aggregation was being impaired. 